\title{
Efectividad de la urtica dioica (ishanga) en el tratamiento de asma infantil
}

\section{Effectiveness of urtica dioica (ishango) in the treatment of childhood asthma in children"}

Ivonne León.

\begin{abstract}
Resumen
Objetivo: Determinar la efectividad de la Urtica Dioica (Ishanga) en el tratamiento de Asma en niños de 2 a 4 años que acuden al Hospital Amazónico Ucayali 2018.

Métodos: El trabajo fue de tipo experimental-prospectivo longitudinal; se trabajó con 2

grupos de muestras (10 niños con asma para el grupo experimental y 10 para el grupo control). Para la recolección de datos se usó: la ficha de asma y la lista de cotejo. Para el análisis descriptivo de los datos estadísticos se realizó el análisis de Chi-cuadrado.

Resultados: Los tipos de asma el mayor porcentaje $40 \%$ de niños del grupo experimental presentaron asma de tipo leve y en el grupo control el 50\%, después de la administración de la Urtica Dioica (Ishanga) el 80\% del grupo experimental no presento ningún tipo de asma y en el grupo control aumentaron de un $50 \%$ a un $60 \%$ de asma en los niños. El análisis estadístico de los datos deja ver que existe una diferencia significativa en la respuesta clínica entre ambos grupos, dado un valor de chi-cuadrado de 3.84 con un valor de $\mathrm{p}=0.05$, con lo cual se acepta la hipótesis alterna que afirma: "La Urtica Dioica (Ishanga) es efectiva para el tratamiento del asma infantil en niños de 2-4 años.

Conclusión: La infusión de Urtica Dioica (Ishanga) en dosis de 100/kg Mg. durante 6 meses reduce el número de exacerbaciones, mejora la función pulmonar a largo plazo y previene el desarrollo de una limitación crónica funcional.
\end{abstract}

Palabras Claves: Urtica Dioica (Ishanga), tratamiento de Asma Infantil.

\begin{abstract}
Objective: To determine the effectiveness of Urtica dioica (ishango) in the treatment of childhood asthma in children 2 to 4 years attending the Support Hospital of Amazonian Ucayali.

Methods: The work was experimental, prospective longitudinal, is working with 2 groups of samples (10 children with asthma for the experimental group and 10 for the control group). For data collection instruments were used: the information about the patient with asthma and checklist. For the descriptive analysis of statistical data analysis was performed chi-square.

Results: With respect to the types of asthma 40\% the highest percentage of children in the experimental group had mild asthma rate in the control group, 50\% after the administration of Urtica dioica (ishango) $80 \%$ of experimental group did not present any type of asthma and the control group increased from $50 \%$ to $60 \%$ of asthma in children. Statistical analysis of the data reveals that there is a significant difference in clinical response between the two groups, given a value of chi-square of 3.84 with a value of $p=0.05$, thus accepts the alternative hypothesis which states: "The Urtica dioica (ishango) is effective for the treatment of childhood asthma in children aged 2-4 years attending the Hospital of Amazonian Ucayali Conclusion: The infusion of Urtica dioica (ishango) in doses of $100 \mathrm{mg} / \mathrm{kg}$. for ten months reduces the number of exacerbations, improved lung function in the long run and prevents the development of chronic functional limitations.
\end{abstract}

Keywords: Urtica dioica (ishango), treatment of childhood asthma.

- Mg. en Salud Publica y Gestión Sanitaria. Dra. En Ciencias de la Salud Docente tiempo Completo UAP i_leon_a@doc.uap.edu.pe 


\section{INTRODUCCIÓN}

El Asma es un Problema de Salud Pública con impacto socioeconómico, se encuentra dentro de las 5 primeras causas de Morbimortalidad infantil. El Perú los casos 2018 es de 6807 casos e incidencia de 24.02. MINSA PERU (1). La mayor incidencia en niños es en Lima. se da entre los meses de mayo y agosto, debido al características climáticas.

Objetivos específicos: Evaluar el Asma Infantil en niños de 2 a 4 años antes de aplicar la Urtica Dioica (Ishanga) en estudio. Manipular y administrar la Urtica dioica (Ishanga) en el tratamiento de Asma Infantil en niños de 2 a 4 años en estudio. Evaluar el asma Infantil en niños de 2 a 4 años después del tratamiento a base de la Urtica Dioica (Ishanga) en estudio. Comprobar la efectividad de la Urtica Dioica (Ishanga) en el tratamiento de Asma Infantil en niños da 2 a 4 años en el estudio.(2)

\section{MATERIAL Y MÉTODO.}

Se realizó en dos grupos de diez niños en el tiempo prospectivo longitudinal con el tipo de variables multivariado. Por las características del estudio, inicialmente se realizó la exploración diagnostica de sintomatología de asma en ambos grupos, el mismo que permitió detectar los problemas y necesidades de tratamiento con Urtica Dioica "Ishanga" en el grupo experimental, el mismo que permitió la intervención para el grupo control.

Finalmente, y luego de la intervención con el grupo experimental, se volvió aplicar posteriormente el instrumento el inicial a ambos grupos para realizar la comparación del tratamiento. Por las características de la investigación la población total de niños con Asma en el año 2018, fue de 109 niños de 2 a 4 años sujeto de estudio la muestra ha sido seleccionada con las características de la muestra probabilística aleatoria simple constituida por 20 niños de dos a cuatro años con diagnóstico médico de asma infantil. Para el presente estudio el diseño de los instrumentos fue formulado teniendo en cuenta los criterios que estar considerados en la estrategia de la etapa niño del MAIS- MINSA Perú a nivel Nacional y del Hospital de Yarinacocha, la Historia clínica pediátrica del paciente con asma y estándares internacionales GINA.(7)

Ficha del paciente asmático, que fue diseñado por la investigadora en el protocolo de Tesis (Ficha del paciente Asmático validado por juicio de expertos). Así como los consentimientos Informados bioéticos pertinentes

La Ishanga fue procesada en laboratorio Fitoquímico para determinar sus componentes medicinales. Para el consumo se deseco en horno microondas común; los tallos y las hojas y pulverizados para ser bebido en infusión en dosis según el peso de cada niño $100 \mathrm{gr} / \mathrm{kg}$ niño. En una toma diaria. Por el tiempo de estudio el cual fue supervisado el grupo control. (3), (4)

\section{En relación a la composición de los ítems está dividido en dos tipos de fichas:}

Ficha para Casos control; contiene en la primera parte datos generales de los pacientes con asma y fueron recolectados mediante una encuesta directa con preguntas cerradas y abiertas y exámenes de diagnóstico general para el asma infantil para determinar la situación real y actual de los diagnósticos, tratamientos, antecedentes medicaciones hospitalizaciones de asma de cada grupo.

Ficha para casos experimental; la segunda parte contiene datos de controles de exámenes físicos y diagnósticos, así como el tratamiento con Urtica Dioca "Ishanga" que fue llenado con entrevista directa al responsable del niño con asma en su domicilio durante una semana de sesión cada mes desde el inicio y hasta finalizar el estudio verificando y supervisando la toma de infusión entregada. La tercera parte comprende datos de exámenes y diagnósticos de asma cada vez acudía al establecimiento de salud para su control con el médico pediatra pos al estudio con los mismos datos de la primera parte. (antes y después). Elaboración de los Datos Se considera las siguientes fases: 
Los Datos fueron revisados, se examinó en forma crítica cada uno de los instrumentos utilizados (control de calidad), a fin de poder hacer las correcciones según el caso y sean pertinentes. Posteriormente estos datos fueron Codificados, se transformó estos datos en códigos numéricos, de acuerdo a la respuesta esperada en el instrumento, según el dominio de la variable. La clasificación de los Datos, se realizó en base a la codificación, escala de medición e indicadores-valoración de cada variable identificada en el estudio. El recuento de datos, fue de acuerdo al método de las matrices de tabulación o cuadros estadísticos de doble entrada rellenados. La presentación de los Datos, se presentó los datos en cuadros de doble entrada y gráficos pertinentes.

\section{RESULTADOS.}

Tabla 1. Niños con asma infantil del grupo experimental y control según edad hospital amazonico, 2018

\begin{tabular}{|l|l|l|l|l|}
\hline \multirow{3}{*}{ EDAD } & \multicolumn{4}{|c|}{ GRUPOS } \\
\cline { 2 - 5 } & EXPERIMENTAL & CONTROL \\
\cline { 2 - 5 } & Fi & $\mathbf{\%}$ & Fi & \% \\
\hline $2-3$ & 4 & 40 & 3 & 30 \\
\hline $3-4$ & 6 & 60 & 7 & 70 \\
\hline TOTAL & $\mathbf{1 0}$ & $\mathbf{1 0 0}$ & $\mathbf{1 0}$ & $\mathbf{1 0 0}$ \\
\hline
\end{tabular}

FUENTE: Ficha de pacientes de Asma.

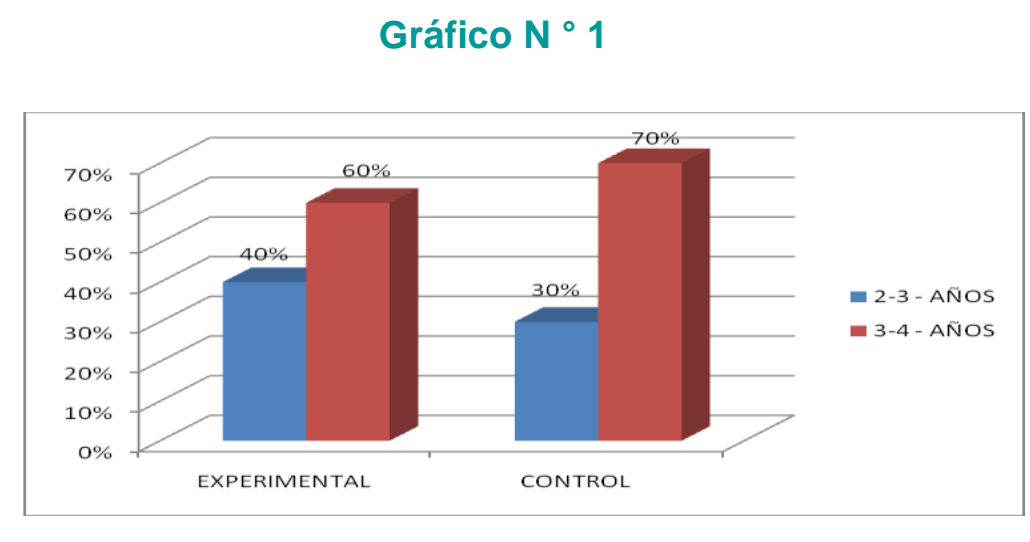

FUENTE: Lista de Cotejo - Ficha de Pacientes de Asma.

\section{Análisis e interpretación:}

En el cuadro y gráfico se observa que, el $60 \%$ (6) de niños del grupo experimental por asma infantil presentaron edades entre 3 - 4 años, el 40\% (4) entre 2-3 años; de los cuales del grupo control, un 70\% (7) presentaron edades entre 3-4 años y, el 30\% (3) entre 2-3 años. 


\begin{tabular}{|l|l|l|l|l|}
\hline \multirow{2}{*}{} & \multicolumn{3}{|l|}{ EXPERIMENTAL } & \multicolumn{2}{l|}{ CONTROL } \\
\cline { 2 - 5 } & Fi & \% & Fi & \% \\
\hline Masculino & 6 & 60 & 5 & 50 \\
\hline Femenino & 4 & 40 & 5 & 50 \\
\hline TOTAL & $\mathbf{1 0}$ & $\mathbf{1 0 0}$ & $\mathbf{1 0}$ & $\mathbf{1 0 0}$ \\
\hline
\end{tabular}

FUENTE: Ficha de pacientes de Asma.

GRÁFICO $\mathbf{N}^{\circ} \mathbf{2}$

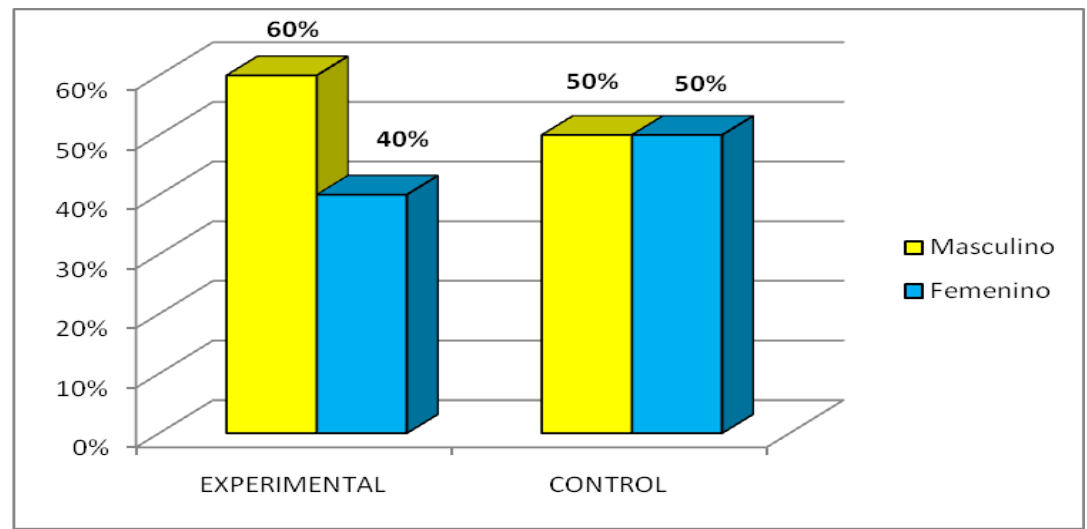

FUENTE: Lista de Cotejo - Ficha de Pacientes de Asma.

\section{ANÁLISIS E INTERPRETACIÓN:}

El cuadro y gráfico nos indica que, el 60\% (6) de niños del grupo experimental con asma infantil pertenecen al sexo masculino y, el $40 \%$ al femenino; por tanto, un 50\% (5) del grupo control pertenecen al sexo masculino y, el 50\% (5) al femenino.

Tabla $\mathrm{N}^{\circ}$ 3. Niños del grupo experimental y control según tipos de asma infantil antes y después del tratamiento a base de la urtica dioica y farmacológico hospital amazonico - 2018

\begin{tabular}{|c|c|c|c|c|c|c|c|c|}
\hline \multirow{2}{*}{ TIPOS DE ASMA } & \multicolumn{9}{|c|}{ GRUPOS EXPERIMENTAL } & \multicolumn{3}{c|}{ GRUPO CONTROL } \\
\cline { 2 - 9 } & ANTES & \multicolumn{1}{c|}{ DESPUÉS } & \multicolumn{2}{c|}{ ANTES } & \multicolumn{2}{c|}{ DESPUÉS } \\
\cline { 2 - 9 } & $\mathbf{F i}$ & $\mathbf{\%}$ & FI & $\mathbf{\%}$ & $\mathbf{F i}$ & $\mathbf{\%}$ & $\mathbf{F i}$ & $\mathbf{\%}$ \\
\hline Leve & 4 & 40 & 0 & 00 & 5 & 50 & 6 & 60 \\
\hline Moderada & 3 & 30 & 1 & 10 & 3 & 30 & 3 & 30 \\
\hline Severa & 3 & 30 & 1 & 10 & 2 & 20 & 1 & 10 \\
\hline Sin Asma & 0 & 00 & 8 & 80 & 0 & 0 & 0 & 00 \\
\hline TOTAL & $\mathbf{1 0}$ & $\mathbf{1 0 0}$ & $\mathbf{1 0}$ & $\mathbf{1 0 0}$ & $\mathbf{1 0}$ & $\mathbf{1 0 0}$ & $\mathbf{1 0}$ & $\mathbf{1 0 0}$ \\
\hline
\end{tabular}


Gráfico n ${ }^{\circ}$ 3. Niños del grupo experimental y control según tipos de asma infantil antes y después del tratamiento a base de la urtica dioica y farmacológico hospital amazonico 2018

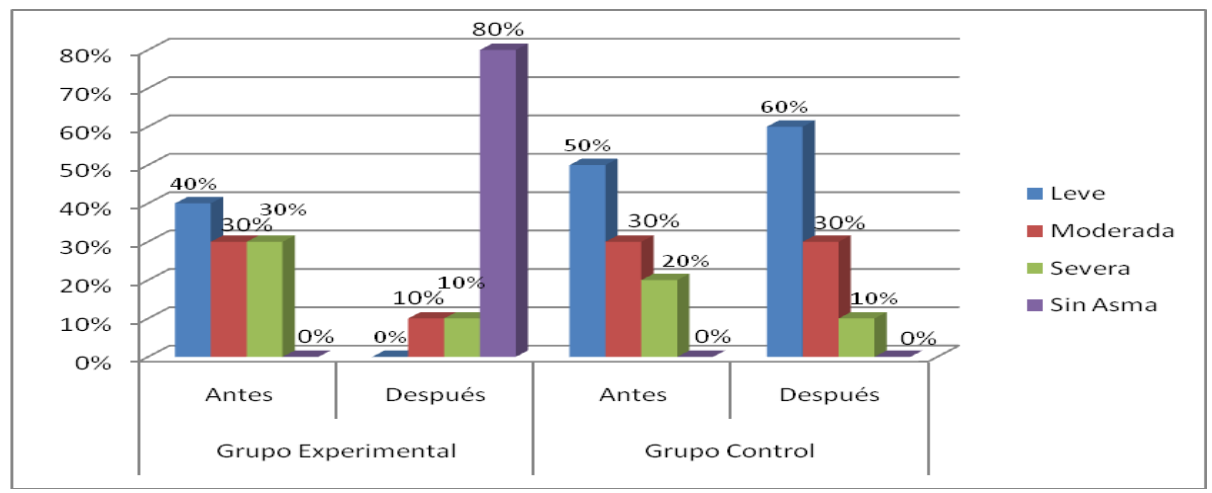

FUENTE: Lista de Cotejo - Ficha de Pacientes de Asma.

\section{ANÁLISIS E INTERPRETACIÓN:}

Es el cuadro y gráfico se observa que, el 100\% (10) niños del grupo experimental presentaron asma antes del tratamiento: leve 40\% (4), moderado 30\% (3) y severo 30\% (3) antes del tratamiento a base de la Urtica Dioica (Ishanga). y, después del tratamiento un $80 \%$ (8) no presentaron el asma infantil. Por tanto, el 100\% (10) de niños del grupo experimental solo 2 con asma $1 \%$ leve $1 \%$ y moderada. Mientras que el grupo control presentaron antes los tipos de asma: leve50\% (5), moderada 30\% (3), severa $2 \%$ antes que reciba el tratamiento farmacológico Hospitalario y, después del tratamiento farmacológico Hospitalario persistía con los tipos de asma Leve 60\% (6) Moderados 30\% (3) y Severo10\% (1) en los niños del grupo control.

\section{DISCUSIÓN}

El trabajo se realizó con la finalidad de confirmar la eficacia del tratamiento del asma con Urtica Dioica (Ishanga). Según los procesos de la investigación realizada los resultados obtenidos fueron: Un porcentaje de $100 \%$ de niños (as) del grupo experimental como del grupo control presentaron los tipos de asma infantil como: Leve; moderado y severo antes del tratamiento a base de la Urtica Dioica (Ishanga) y farmacológica hospitalaria. Por tanto, la Organización Mundial de la Salud afirma: La palabra asma se origina de una antigua palabra griega que significa jadeante. Esencialmente, el asma es una incapacidad de respirar adecuadamente; cuando una persona inhala, el aire pasa por los pulmones mediante las vías respiratorias progresivamente más pequeñas llamadas los bronquiolos. La Organización Panamericana de la Salud (OPS) señala: Los mecanismos que causan asma son complejos y varían entre grupos de población y aun entre individuos.(5), (6).

El asma es peor durante las horas de noche y los ataques ocurren a menudo entre 2.00 a. m. a 4:00 a.m. por varias razones: los cambios químicos y la temperatura corporal causan inflamación y el reducimiento de las vías respiratorias; respuestas alérgicas retardadas pueden ocurrir a causa de la exposición a los alérgenos durante el día; hacia la madrugada, el efecto de los medicamentos inhala torios pueden desaparecer y desencadenar un ataque asmático de diverso grado. 
Desde un punto de vista, en el estudio encontramos que un promedio de $80 \%$ de niños del grupo experimental con asma infantil, fueron solucionados los problemas del asma, leve, moderado y severo a través del proceso de la manipulación y explicación de la Urtica Dioica (Ishanga), a diferencia del grupo control que seguían presentando dichos tipos de asma que fueron tratados farmacológicamente según GINA.(7) Lo cierto, es que la planta Urtica Dioica (Ishanga), tiene un efecto curativo para el asma infantil, donde se evaluó nuestra muestra de estudio dando un resultado positivo. Donde el estudio de niño(a) asmático (a) se realizó con base en un historial personal y familiar, exámenes de laboratorio, radiografías de tórax y pruebas de función pulmonar.

Las exacerbaciones y episodios agudos de las enfermedades se asocian con la exposición a factores ambientales, como: Humo de la Cocina, abuelos fumadores de tabaco sin procesar y factores propios de la región con clima tropical Amazónico y húmedo y que pueden determinar un patrón comportamiento de la enfermedad ya determinado. No obstante, pueden acompañarse de estertores debido a la abundancia de secreciones que el niño pequeño no consigue expectorar. (8)La Urtica Dioica (Ishanga) es usada en enfermedades respiratorias agudas y Crónicas. González Daga afirma en su libro de Homeopatía de tratamiento Naturales para el asma, que Urtica Dioica (Ishanga) se encuentra dentro de los remedios naturales que se provee para el asma y secreciones bronquiales, es fundamentalmente, expectorante fluidificaste bronquial, calma los espasmos de los bronquios y ayuda a liberar las secreciones pulmonares, así como descongestionante y antiflamatorio.es utilizado como remedio natural para combatir el asma.(9)

Es indudable que en nuestro estudio realizado y comprobado según la contratación de la hipótesis llegamos al resultado obtenido que Urtica Dioica (Ishanga) es efectiva para el tratamiento del Asma Infantil entre niños de 2 a 4 años, Resultado obtenidos a través de la manipulación y aplicaron de dicha planta herbácea.

\section{CONCLUSIONES}

\section{Durante el proceso de la investigación realizada se llegaron a las conclusiones siguientes:}

Un creciente número de niños con asma infantil estudiados del grupo experimental el $60 \%$ representaron entre 3 a 4 años y el 70\% del grupo control. Por otro lado, un $60 \%$ fueron del sexo masculino del grupo experimental y un promedio de $50 \%$ dividido entre el masculino y el femenino del grupo control.

Un promedio del porcentaje de 70\% de niños del grupo experimental presentaron asma leve aplicación de la Urtica Dioica (Ishanga) y no llegando a moderada, ni crisis asmáticas y el grupo.

Después de la aplicación de la planta Urtica Dioica (Ishanga) el grupo experimental 80\% no presentaron ningún tipo de asma infantil, a diferencia del grupo control que, si presentaron 100\%, debido a que no se le aplico Urtica Dioica (Ishanga).(10)

A través de la contrastación y prueba estadística de la hipótesis se llegaron a aceptar la siguiente afirmación: 
Urtica Dioica (Ishanga) fue efectiva para el tratamiento de asma infantil en niños de 2 a 4 años. Según la contratación de la hipótesis se llegó a aceptar el resultado Pt.= 3.8416 .4 H

Se concluye que la de Infusión de Urtica Dioica (Ishanga) en dosis de $100 \mathrm{Mg} / \mathrm{kg}$ en infusión. durante un largo período de seguimiento de niños asmáticos, además de carecer de efectos secundarios, reduce el número de exacerbaciones, mejora la función pulmonar a largo plazo y previene el desarrollo de una limitación crónica funcional. Estos beneficios son superiores a los conseguidos con otras terapias como farmacológicas que tienen efectos secundarios en los niños menores de 5 años. (11)

\section{REFERENCIAS}

1. MINSA PERU OGE /2018 ASIS

2. Chatkin MN, Meneses AMB. Prevalencia e factores de riesgo para asma en escolares de una cohorte de Sul do Brasil. J Pediatría (Rio J). 2005; 81(5): 411- 446.

3. Carballo A. La evaluación preclínica y el ensayo clínico de plantas medicinales, rev. Medicentro Santa clara Cuba.

4. Cortijo J. Modelos experimentales de asma. Aportaciones y limitaciones. Arch. Bronconeumol. 2003; 39:54-6.

5. Fototerapia: Vademécum de prescripción de plantas medicinales. Asociación Española de Médicos Naturistas y Colegio Oficial de Farmacéuticos de Vizcaya. 2001; 4: 34-56.

6. Guía de las plantas medicinales. 2008; 23-35.

7. Global Initiative for Asthma (GINA). Global Strategy for Asthma Management and prevention. Bethesda MD: National Institutes of Health, National Heart, Lung and Blood; 2002.

8. Harrison. Principios de Medicina Interna. 14ª edición. Editorial Mcgraw Gill. Madrid, España. 1998; 134-147.

9. Heaton T, Rowe J, Turner S. Respirar. El Portal sobre asma en niños y adolescentes 2005.

10. J.L. Diez Jarilla.- Problemas Clínicos en Neumología Pediátrica. 200; 101-125.

11. Kosmann, Ingrid, Vicente, Carlos A.- Sanarnos con Plantas Iquitos Perú 2009.

Correo de contacto:

\section{i_leon_a@doc.uap.edu.pe}

